\title{
Impact of rotation and disc lifetime on pre-main sequence lithium depletion of solar-type stars
}

\author{
P. Eggenberger, L. Haemmerlé, G. Meynet, and A. Maeder \\ Observatoire de Genève, Université de Genève, 51 Ch. des Maillettes, 1290 Sauverny, Suisse \\ e-mail: [patrick.eggenberger; lionel.haemmerle; georges.meynet; andre.maeder]@unige.ch
}

Received 10 November 2011 / Accepted 4 January 2012

\begin{abstract}
Aims. We study the influence of rotation and disc lifetime on lithium depletion of pre-main sequence (PMS) solar-type stars. Methods. The impact of rotational mixing and of the hydrostatic effects of rotation on lithium abundances are investigated by computing non-rotating and rotating PMS models that include a comprehensive treatment of shellular rotation. The influence of the disc lifetime is then studied by comparing the lithium content of PMS rotating models experiencing different durations of the disc-locking phase between 3 and $9 \mathrm{Myr}$.

Results. The surface lithium abundance at the end of the PMS is decreased when rotational effects are included. During the beginning of the lithium depletion phase, only hydrostatic effects of rotation are at work. This results in a decrease in the lithium depletion rate for rotating models compared to non-rotating ones. When the convective envelope recedes from the stellar centre, rotational mixing begins to play an important role due to differential rotation near the bottom of the convective envelope. This mixing results in a decrease in the surface lithium abundance with a limited contribution from hydrostatic effects of rotation, which favours lithium depletion during the second part of the PMS evolution. The impact of rotation on PMS lithium depletion is also found to be sensitive to the duration of the disc-locking phase. When the disc lifetime increases, the PMS lithium abundance of a solar-type star decreases owing to the higher efficiency of rotational mixing in the radiative zone. A relationship between the surface rotation and lithium abundance at the end of the PMS is then obtained: slow rotators on the zero-age main sequence are predicted to be more lithium-depleted than fast rotators due to the increase in the disc lifetime.
\end{abstract}

Key words. stars: rotation - stars: pre-main sequence - stars: abundances

\section{Introduction}

Rotation is one of the key processes to influence all outputs of stellar models (see e.g. Maeder 2009). While rotational effects have an especially strong impact on the physics and evolution of massive stars, they can also change the global properties and evolution of solar-type stars by counteracting the effets of atomic diffusion and bringing fresh hydrogen fuel to the central stellar core (e.g. Eggenberger et al. 2010b). Observations of light element abundances bring valuable constraints to our modelling of these transport processes (e.g. Pinsonneault 2010). In a preceding work (Eggenberger et al. 2010a), we investigated the influence of shellular rotation on the lithium abundances of main-sequence solar-type stars in the context of a possible link between lithium depletion and the rotational history of exoplanet-host stars proposed by Bouvier (2008). We then found that the larger efficiency of rotational mixing predicted in exoplanet-host stars can result in lower lithium abundances for these stars during the main sequence. After this study of the impact of shellular rotation on the lithium abundances of mainsequence stars, we are now interested in investigating the effects of rotation on the lithium content of pre-main sequence (PMS) solar-type stars.

The impact of rotation on PMS lithium depletion of solartype stars has already been discussed (e.g. Pinsonneault et al. 1990; Martin \& Claret 1996; Mendes et al. 1999). These previous studies focused mainly on the hydrostatic effects of rotation $^{1}$ and did not consider the influence of a possible coupling between the star and its disc. The star-disc interaction plays a key role in determining the rotation of the star on the zero-age main sequence (ZAMS). By assuming that the presence of the disc prevents the star from spinning up so that its surface angular velocity remains constant, one finds that the influence of this coupling simply relies on the disc lifetime. A longer disc lifetime then enables the star to lose a larger amount of angular momentum during the PMS, resulting in lower rotation rates on the ZAMS. In addition to its effect on the surface rotational velocity during the PMS, the duration of the disc-locking phase can change the internal rotation profiles, with a possible impact on the lithium content. In this paper, we thus investigate the influence of rotation and disc lifetime on the PMS lithium depletion of solar-type stars. Both effects of rotational mixing and of the centrifugal force are studied by computing models that include a comprehensive treatment of shellular rotation. The effects of the disc lifetime on the PMS lithium depletion are then investigated by comparing the PMS evolution of models with different durations of the disc-locking phase.

The modelling of rotation is first briefly described in Sect. 2 . The effects of rotation on PMS lithium depletion are discussed in Sect. 3, while the impact of the disc lifetime is investigated in Sect. 4. The conclusion is given in Sect. 5 .

\footnotetext{
${ }^{1}$ By hydrostatic effects of rotation, we mean here the effects related to the change in the effective gravity due to rotation (the effective gravity being defined as the classical gravity diminished by the centrifugal acceleration).
} 


\section{Modelling rotation}

The stellar evolution code used for these computations is the Geneva code, which includes a detailed treatment of shellular rotation (Eggenberger et al. 2008). In the context of shellular rotation, the transport of angular momentum in radiative zones obeys an advection-diffusion equation written in Lagrangian coordinates (Zahn 1992; Maeder \& Zahn 1998):

$\rho \frac{\mathrm{d}}{\mathrm{d} t}\left(r^{2} \Omega\right)_{M_{r}}=\frac{1}{5 r^{2}} \frac{\partial}{\partial r}\left(\rho r^{4} \Omega U(r)\right)+\frac{1}{r^{2}} \frac{\partial}{\partial r}\left(\rho D r^{4} \frac{\partial \Omega}{\partial r}\right)$,

where $r$ is a characteristic radius, $\rho$ the mean density on an isobar, and $\Omega(r)$ the mean angular velocity at level $r$. The vertical component $u(r, \theta)$ of the velocity of the meridional circulation at a distance $r$ to the centre and at a colatitude $\theta$ can be written

$u(r, \theta)=U(r) P_{2}(\cos \theta)$.

Only the radial term $U(r)$ appears in Eq. (1). Its expression is given by (Maeder \& Zahn 1998)

$$
U(r)=\frac{P}{\rho g C_{P} T\left[\nabla_{\mathrm{ad}}-\nabla+(\varphi / \delta) \nabla_{\mu}\right]}\left\{\frac{L}{M}\left(E_{\Omega}+E_{\mu}\right)\right\},
$$

where $P$ is the pressure, $C_{P}$ the specific heat, $E_{\Omega}$ and $E_{\mu}$ are terms depending on the $\Omega$ - and $\mu$-distributions respectively, up to the third-order derivatives and on various thermodynamic quantities (see Maeder \& Zahn 1998, for more details).

Meridional circulation and shear mixing are considered as the main mixing mechanisms in radiative zones. In convective zones, solid-body rotation is assumed as indicated by the solar case. The first term on the right-hand side of Eq. (1) describes the advection of angular momentum by meridional circulation, while the second term accounts for the transport of angular momentum by shear instability with $D=D_{\text {shear }}$. The expression of this diffusion coefficient is given by

$$
\begin{aligned}
D_{\text {shear }}= & \frac{4\left(K+D_{\mathrm{h}}\right)}{\left[\frac{\varphi}{\delta} \nabla_{\mu}\left(1+\frac{K}{D_{\mathrm{h}}}\right)+\left(\nabla_{\mathrm{ad}}-\nabla_{\mathrm{rad}}\right)\right]} \\
& \times \frac{H_{\mathrm{p}}}{g \delta} f_{\text {energ }}\left(\frac{9 \pi}{32} \Omega \frac{\mathrm{d} \ln \Omega}{\mathrm{d} \ln r}\right)^{2},
\end{aligned}
$$

with $K$ the thermal diffusivity, $f_{\text {energ }}$ the fraction of the excess energy in the shear that contributes to mixing (here taken equal to 1) (Maeder \& Meynet 2001), and $D_{\mathrm{h}}$ the diffusion coefficient associated to horizontal turbulence. The usual expression for this coefficient is, according to Zahn (1992),

$D_{\mathrm{h}}=\frac{1}{c_{\mathrm{h}}} r|2 V(r)-\alpha U(r)|$,

where $U(r)$ is the vertical component of the meridional circulation velocity, $V(r)$ the horizontal component, $c_{\mathrm{h}}$ a constant of order unity (here taken equal to 1) and $\alpha=\frac{1}{2} \frac{\mathrm{d} \ln r^{2} \bar{\Omega}}{\mathrm{d} \ln r}$. The full solution of Eq. (1) taking $U(r)$ and $D$ into account gives the nonstationary solution of the problem. We recall here that meridional circulation is treated as a truly advective process in the Geneva evolution code.

The vertical transport of chemicals through the combined action of vertical advection and strong horizontal diffusion can be described as a pure diffusive process (Chaboyer \& Zahn 1992). The advective transport is thus replaced by a diffusive term, with an effective diffusion coefficient

$D_{\text {eff }}=\frac{|r U(r)|^{2}}{30 D_{\mathrm{h}}}$.
The vertical transport of chemical elements then obeys a diffusion equation that, in addition to this macroscopic transport, also accounts for (vertical) turbulent transport:

$$
\frac{\mathrm{d} X_{i}}{\mathrm{~d} t}=\dot{X}_{i}+\frac{1}{\rho r^{2}} \frac{\partial}{\partial r}\left[r^{2} \rho\left(D_{\mathrm{eff}}+D_{\text {shear }}\right) \frac{\partial X_{i}}{\partial r}\right],
$$

where $X_{i}$ is the mass fraction of element $i$, and $\dot{X}_{i}$ represents the variations in chemical composition due to nuclear reactions.

\section{Effects of rotation}

To study the effects of rotation on the lithium abundances of PMS solar-type stars, we compute the PMS evolution of $1 M_{\odot}$ models with a solar chemical composition as given by Grevesse \& Noels (1993) and a solar-calibrated value for the mixinglength parameter. One model is first computed without rotation. The corresponding rotating model shares exactly the same initial parameters except for including a comprehensive treatment of shellular rotation. This model is computed with an initial angular velocity of $20 \Omega_{\odot}$ and a disc lifetime of 6 Myr. During the disclocking phase, the surface angular velocity of the star is simply assumed to remain constant.

The evolutionary tracks in the HR diagram are shown for both models in Fig. 1. Including rotation shifts the track to slightly lower effective temperatures and luminosities as previously found by many studies (see e.g. Pinsonneault et al. 1989; Martin \& Claret 1996; Mendes et al. 1999). Figure 2 compares the evolution of the surface lithium abundance during the PMS for the models computed with and without rotation starting with a lithium abundance $A(\mathrm{Li})=\log [N(\mathrm{Li}) / N(H)]+12=3.26$ (Asplund et al. 2009). At the end of the PMS, the rotating model exhibits a lower surface lithium abundance than the non-rotating one (difference of about 0.1 dex for a total lithium depletion of about 1 dex). We thus see that the inclusion of rotation results in a global increase of the lithium depletion during the PMS.

To investigate whether these differences in the lithium content between rotating and non-rotating models are mainly due to rotational mixing or to hydrostatic effects of rotation, another rotating model of $1 M_{\odot}$ is computed by only accounting for the effects of rotational mixing. The initial rotational velocity and disc lifetime of this model are thus identical as for the rotating model including the full treatment of shellular rotation, but the effects of the centrifugal force on the stellar structure are not taken into account. The evolution of this model in the HR diagram is also shown in Fig. 1, but the corresponding track is superimposed on the one of the non-rotating model. The change in the evolutionary tracks in the HR diagram induced by rotation for PMS solar-type stars is thus mainly due to the effects of the centrifugal force and not to a change in the global properties induced by rotational mixing.

Concerning the evolution of the lithium content during the PMS, the situation is quite different from the changes observed in the HR diagram. Figure 2 indeed shows that the lithium depletion is greater for the rotating model including only the effects of rotational mixing than for the non-rotating model. While the effects of rotation on the PMS evolutionary tracks in the HR diagram are mainly due to the centrifugal force, rotational mixing is found to play an important role in the increase of lithium depletion for rotating models during the PMS. As seen in Fig. 2, both rotating models exhibit lower lithium surface abundances than the non-rotating model on the ZAMS. We also note that the lithium depletion occurs earlier for the rotating model, when accounting only for the effects of rotational mixing, than for the 


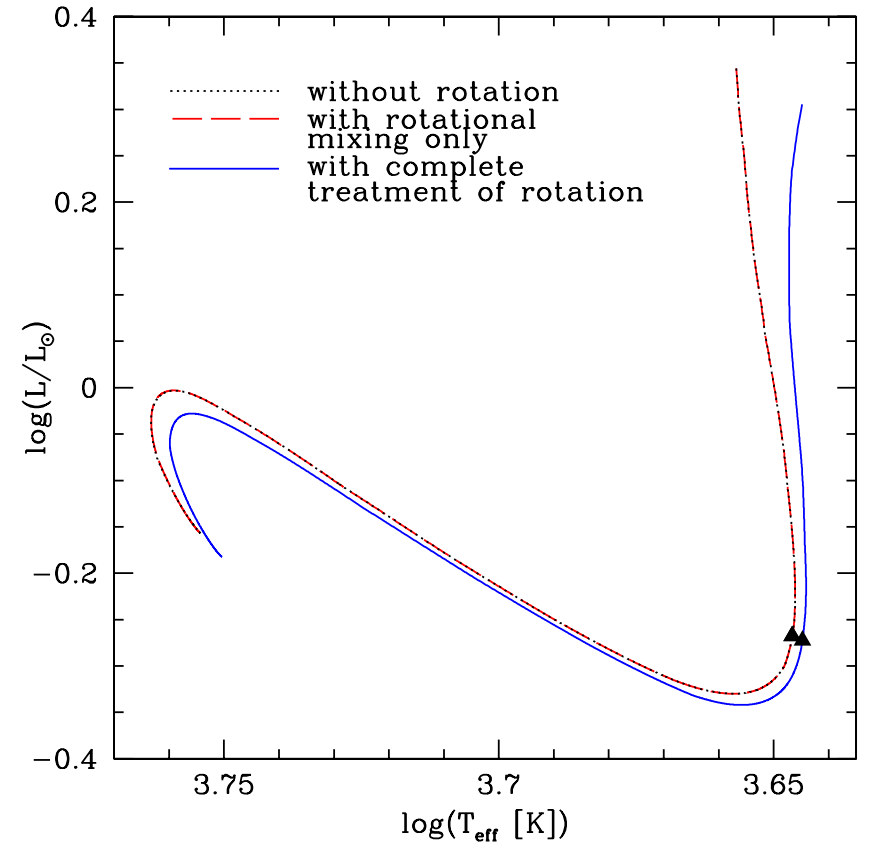

Fig. 1. Effects of rotation on the PMS evolutionary tracks in the HR diagram for $1 M_{\odot}$ models. The dotted and continuous lines correspond to a non-rotating model and a rotating model computed with a full treatment of shellular rotation, respectively. The track corresponding to a rotating model including only the effects of rotational mixing is shown by a dashed line and is superimposed on the track of the non-rotating model. The tracks end when the ZAMS is reached; the end of the disc-locking phase is indicated by a triangle.

model that includes the full treatment of rotational effects. This is related to the hydrostatics effects of rotation. Rotational mixing has a negligible impact on the global stellar structure during the PMS, so that the temperature profile, the depth of the convective envelope, and the evolutionary tracks in the HR diagram are identical for the non-rotating model and the rotating model computed without the hydrostatics effects of rotation. For the model including a complete treatment of rotation, the temperature at the base of the convective envelope is lower during the initial phase when the star is still fully convective. This is caused by the effective gravity of the star being reduced by the centrifugal acceleration term, and this results in a lower lithium depletion rate. Including the centrifugal force therefore leads to a higher lithium content during the beginning of the lithium depletion phase on the PMS.

After this initial phase, a radiative core begins to develop at the centre of the star. The temperature at the base of the convective envelope is slightly higher for the model that includes a complete treatment of rotation than for the non-rotating one during the end of the PMS evolution. After decreasing the lithium depletion rate during the beginning of the PMS, the hydrostatic effects of rotation are thus found to favour lithium depletion during the second part of the PMS, in good agreement with the study by Mendes et al. (1999). More importantly, rotational mixing begins to play a dominant role during this phase. As can be seen in Fig. 2 by comparing the non-rotating model to the model that includes rotational mixing only, the impact of rotational mixing on the surface lithium abundance begins to be visible after about $3 \mathrm{Myr}$. By transporting lithium to deeper layers where it is easily destroyed, such mixing generates an increase in the lithium depletion. In the present PMS models, shear mixing

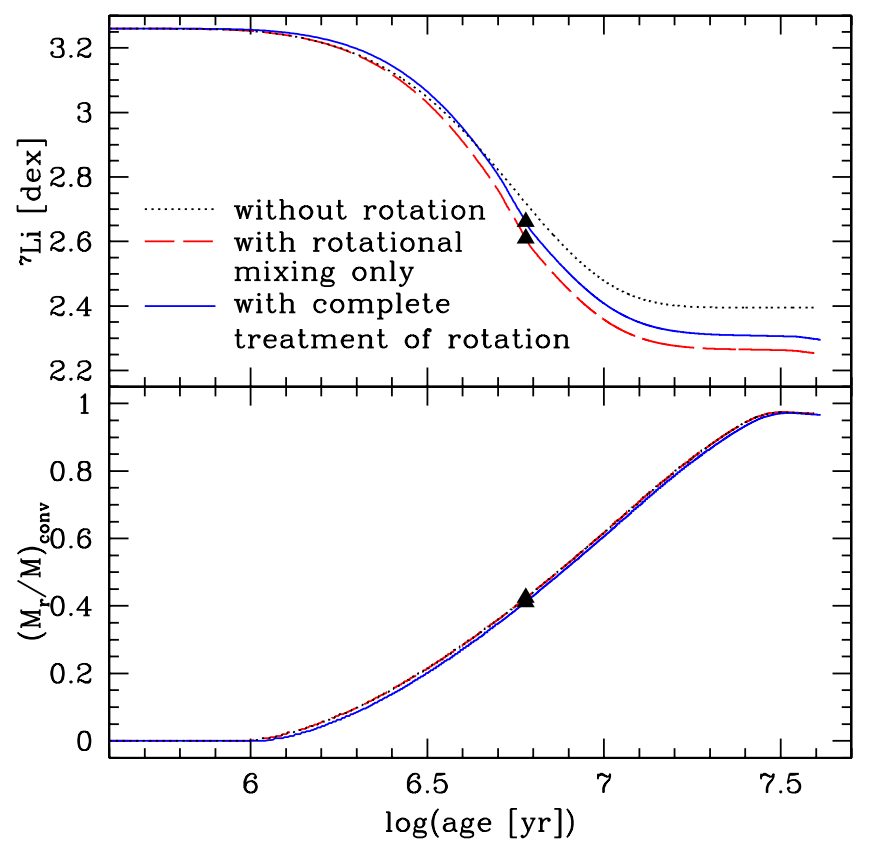

Fig. 2. Top: effects of rotation on lithium depletion during the PMS for $1 M_{\odot}$ models. The non-rotating model is indicated by the dotted line, while the dashed and continuous lines correspond to rotating models that only include the effects of rotational mixing and a full treatment of shellular rotation, respectively. These rotating models are computed with the same initial angular velocity and disc lifetime. The tracks end when the ZAMS is reached and a triangle indicates the end of the disclocking phase. Bottom: location of the mass coordinate of the base of the convective envelope. The track corresponding to a rotating model that only includes the effects of rotational mixing is superimposed on the track of the non-rotating model.

plays the dominant role for lithium depletion with a negligible contribution from meridional currents. The decrease in the surface lithium abundance by rotational mixing is thus directly related to the increase of differential rotation in the stellar interior during the PMS evolution.

Figure 3 shows the internal rotation profile at different ages for the rotating model including a complete treatment of rotational effects. At the beginning of the PMS evolution, the star is fully convective and rotates as a solid body. As soon as the radiative core develops, differential rotation takes place in the radiative zone mainly as a result of the star contracting. During the disc lifetime, the star is assumed to be locked to its disc with a constant surface angular velocity. As soon as the disc disappears, the angular velocity at the stellar surface increases as shown in Fig. 3. For the present model computed with a disc lifetime of $6 \mathrm{Myr}$, the angular velocity at the surface thus remains constant during the first $6 \mathrm{Myr}$. Differential rotation in the radiative zone is enhanced by disc locking and leads to a steep rotation profile at the base of the convective envelope. This generates turbulence below the convective envelope through shear instability, and the resulting mixing can then transport lithium to deeper and hotter regions, where it is destroyed more efficiently.

The quantitative impact of rotation on the PMS lithium content of solar-type stars is of course sensitive to the initial angular velocity adopted when the star is fully convective. To illustrate this point, two additional models of $1 M_{\odot}$ are computed with initial angular velocities of 5 and $10 \Omega_{\odot}$. These models are also computed with a solar chemical composition, a solar-calibrated 


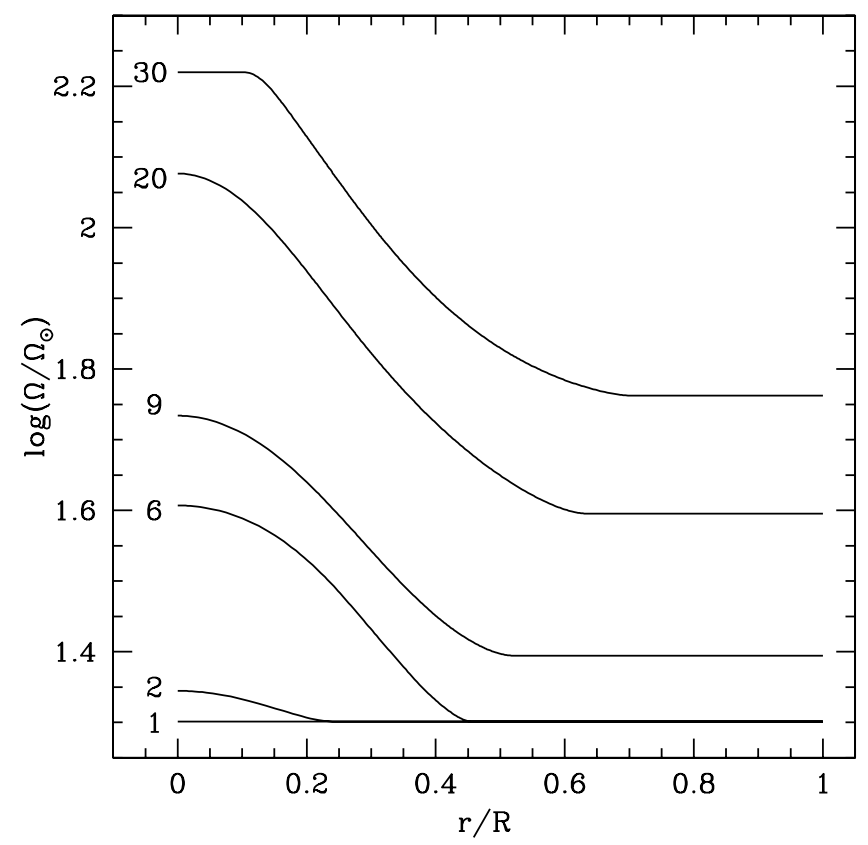

Fig. 3. Evolution of the internal rotation profile for a $1 M_{\odot}$ rotating model with a disc lifetime of $6 \mathrm{Myr}$. The age in $\mathrm{Myr}$ is indicated for each rotation profile.

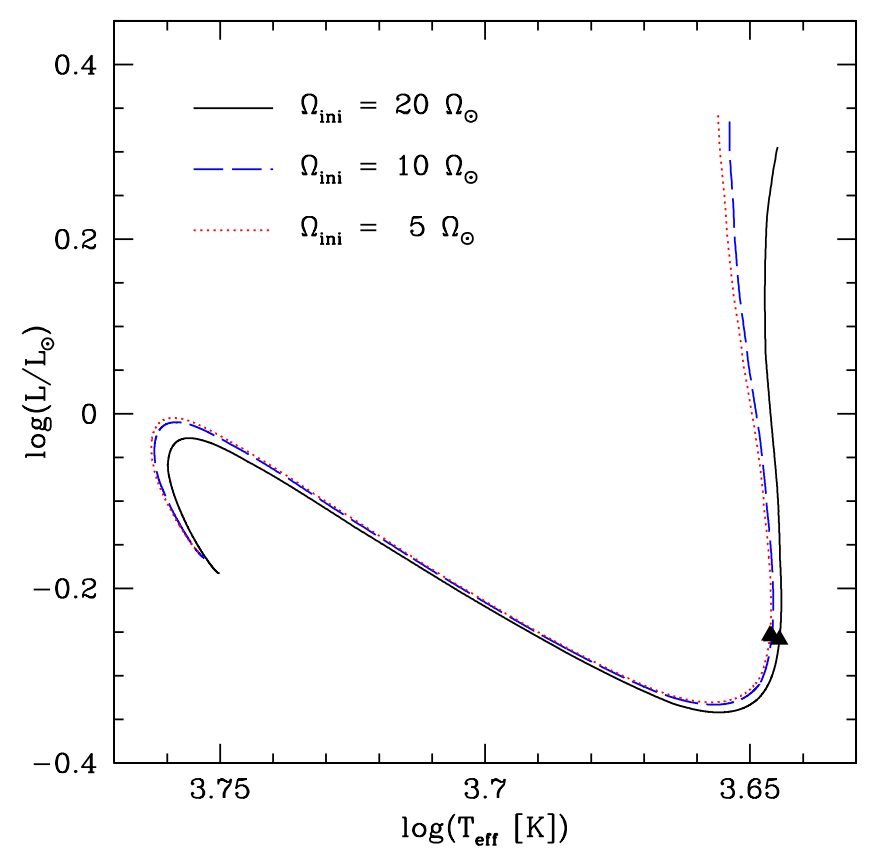

Fig. 4. Evolutionary tracks in the HR diagram for rotating PMS models of $1 M_{\odot}$. The continuous, dashed, and dotted lines correspond to initial velocities of $20 \Omega_{\odot}, 10 \Omega_{\odot}$, and $5 \Omega_{\odot}$, respectively. The tracks end when the ZAMS is reached and the end of the disc-locking phase is indicated by a triangle.

value for the mixing-length parameter and a disc lifetime of 6 Myr. Figure 4 shows the evolutionary tracks in the HR diagram of these models together with the $20 \Omega_{\odot}$ model discussed before. The evolution of the surface lithium abundance of these models is given in Fig. 5. The decrease in the lithium abundance at the end of the PMS is found to be more pronounced for

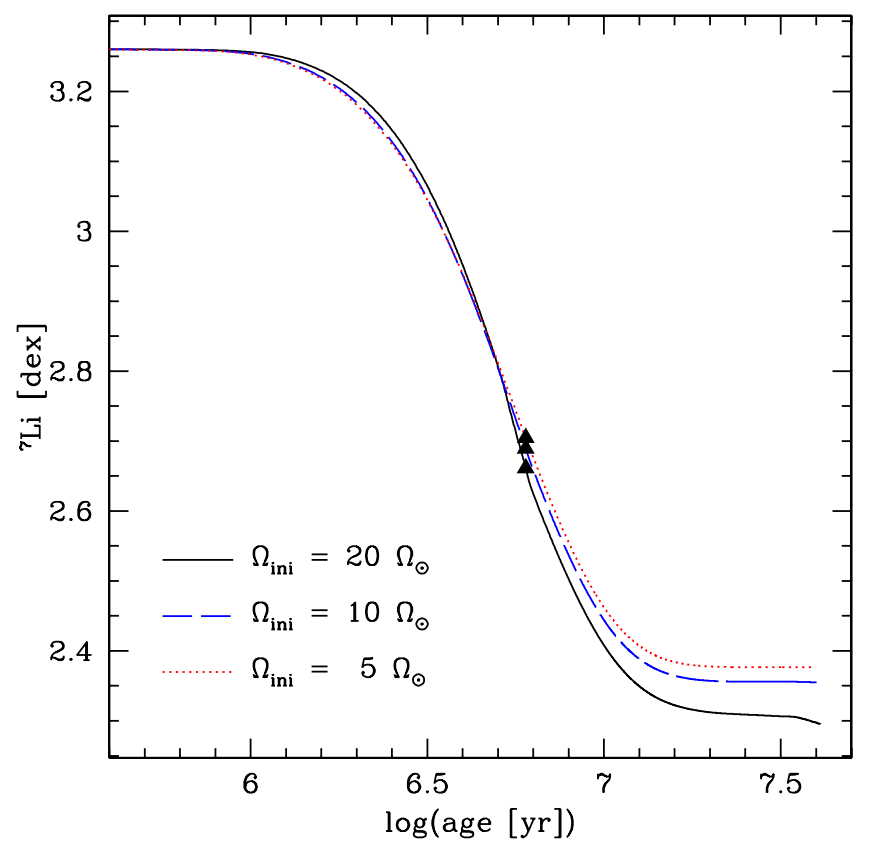

Fig. 5. Lithium surface abundance during the PMS for rotating $1 M_{\odot}$ models. The continuous, dashed, and dotted lines correspond to initial velocities of $20 \Omega_{\odot}, 10 \Omega_{\odot}$, and $5 \Omega_{\odot}$, respectively. The triangles indicate the end of the disc-locking phase. The tracks end when the ZAMS is reached.

higher initial velocities. This mainly results from the increased efficiency of rotational mixing when the rotation rates increase. The hydrostatic effects of rotation are also increased when the initial angular velocity is higher. As shown in Fig. 4, the shift to lower temperatures and luminosities becomes more visible in the HR diagram when the initial rotational velocity increases. This leads to a slight decrease in the lithium depletion rate at the beginning of the PMS evolution when the initial velocity increases. As seen in Fig. 5, lithium depletion therefore occurs earlier for lower initial rotation velocities.

\section{Effects of disc lifetime}

In the preceding section, the effects of rotation on the PMS lithium depletion of solar-type stars have been discussed by comparing rotating models computed with the same disc lifetime of 6 Myr. We are now interested in investigating the impact of the disc lifetime on the lithium depletion during the PMS. Rotating models of $1 M_{\odot}$ are computed for different disc lifetimes of 3-9 Myr. All these models are computed with the same initial angular velocity of $20 \Omega_{\odot}$, a solar chemical composition, and a solar-calibrated value for the mixing-length parameter.

The evolution of the surface angular velocity during the PMS is shown in Fig. 6 for these models. The angular velocity at the surface remains constant during the disc-locking phase. As soon as the disc disappears, the surface angular velocity increases as a result of the stellar contraction until the ZAMS is reached. When the disc lifetime increases, the star keeps its inital surface angular velocity for a longer time and loses a larger amount of angular momentum. As shown in Fig. 6, the increased surface angular velocity then occurs later for longer disc lifetimes, resulting in lower rotational velocities on the ZAMS.

Figure 7 shows the evolutionary tracks in the HR diagram corresponding to these rotating models computed for various 


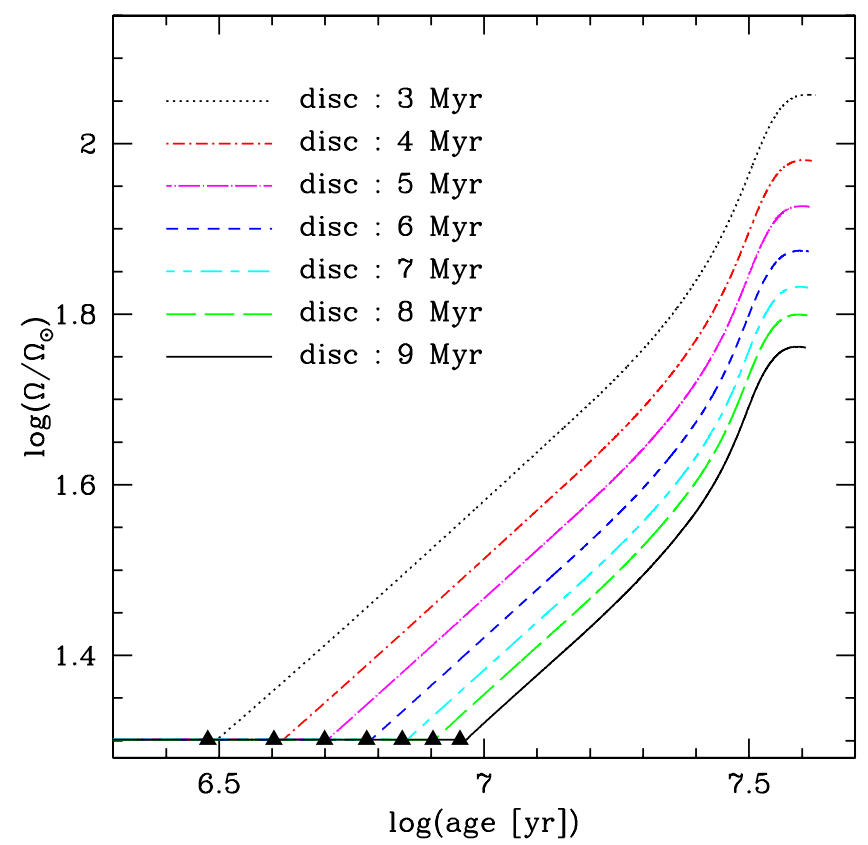

Fig. 6. Evolution of the surface angular velocity for $1 M_{\odot}$ models sharing the same initial velocity but computed with different disc lifetimes. Triangles indicate the end of the disc-locking phase for each model. The tracks end when the ZAMS is reached.

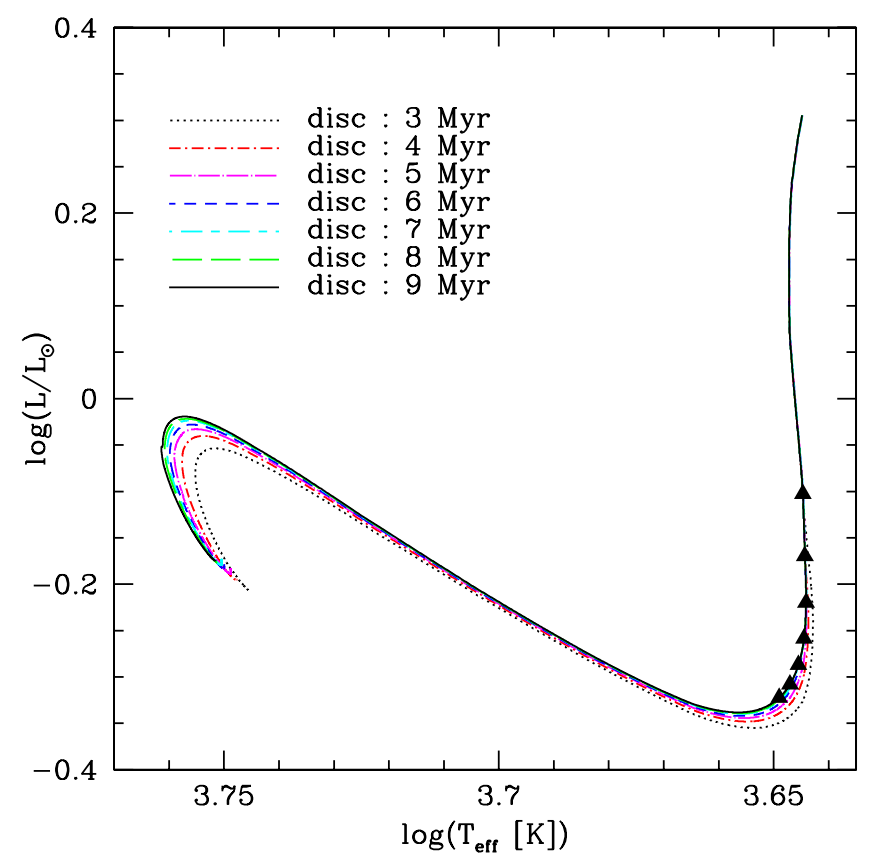

Fig. 7. Evolutionary tracks in the HR diagram for $1 M_{\odot}$ models with the same initial velocity but different disc lifetimes. Triangles indicate the end of the disc-locking phase for each model.

durations of the disc-locking phase. During the first $3 \mathrm{Myr}$, all models follow the same track in the HR diagram because they share the same rotational history. Rotating models with shorter disc lifetimes exhibit higher rotational velocities as soon as the disc disappears, compared to rotating models that remain coupled to their disc for a longer time. Consequently, the hydrostatic effects of rotation are more pronounced for shorter disc

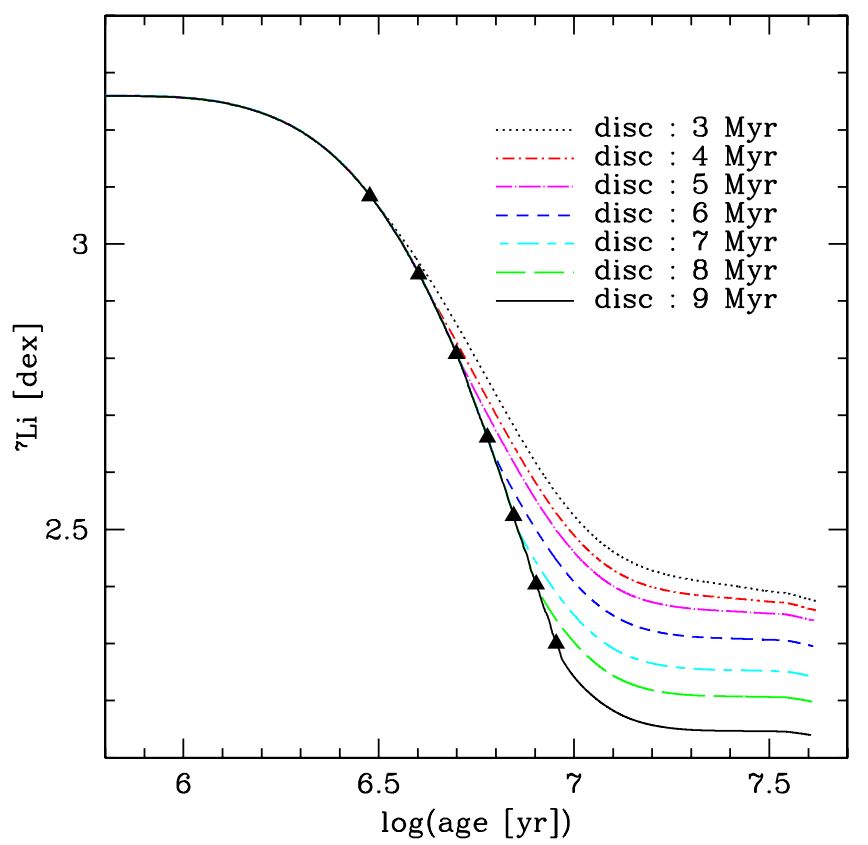

Fig. 8. Evolution of the surface lithium abundance for $1 M_{\odot}$ models with the same initial velocity but different disc lifetimes. Triangles indicate the end of the disc-locking phase for each model.

lifetimes; this results in larger shifts to the red part of the HR diagram when the disc lifetime decreases.

The evolution of the surface lithium abundance is shown in Fig. 8. While the effects of rotation in the HR diagram are found to be stronger for models with shorter disc lifetimes, we see exactly the opposite for the impact of disc lifetime on lithium depletion. Figure 8 indeed shows that lithium depletion during the PMS increases for longer durations of the disc-locking phase. Soon after the disappearance of the disc, the lithium depletion decreases compared to models with longer disc lifetimes. As a result, models computed with shorter disc lifetimes exhibit higher lithium abundances on the ZAMS than models experiencing a longer disc-locking phase.

Figure 9 shows the internal rotation profiles of these models with different disc lifetimes at an age of $9 \mathrm{Myr}$, which corresponds to the end of the disc-locking phase for the model with the longer disc lifetime. The gradient of angular velocity below the convective envelope increases when the disc lifetime increases. Rotational mixing is then more efficient in the interiors of models with longer disc lifetimes, which explains the increased lithium depletion shown in Fig. 8. We thus conclude that an increase of the disc lifetime leads to a decrease in the lithium content during the PMS evolution of a solar-type star owing to the larger amount of differential rotation generated during the disc-locking phase.

The PMS lithium depletion predicted by non-rotating solar calibrated models is known to be too high to correctly reproduce the observations in open clusters (e.g. D'Antona \& Mazzitelli 1994; Ventura et al. 1998; Schlattl \& Weiss 1999; Piau \& Turck-Chièze 2002; D’Antona \& Montalbán 2003). Since including rotational effects results in lower lithium abundances for rotating models compared to non-rotating ones (with a higher impact for longer disc lifetimes), rotation does not solve this problem. One possibility for correctly reproducing the lithium abundances observed in open clusters is to reduce the value of 


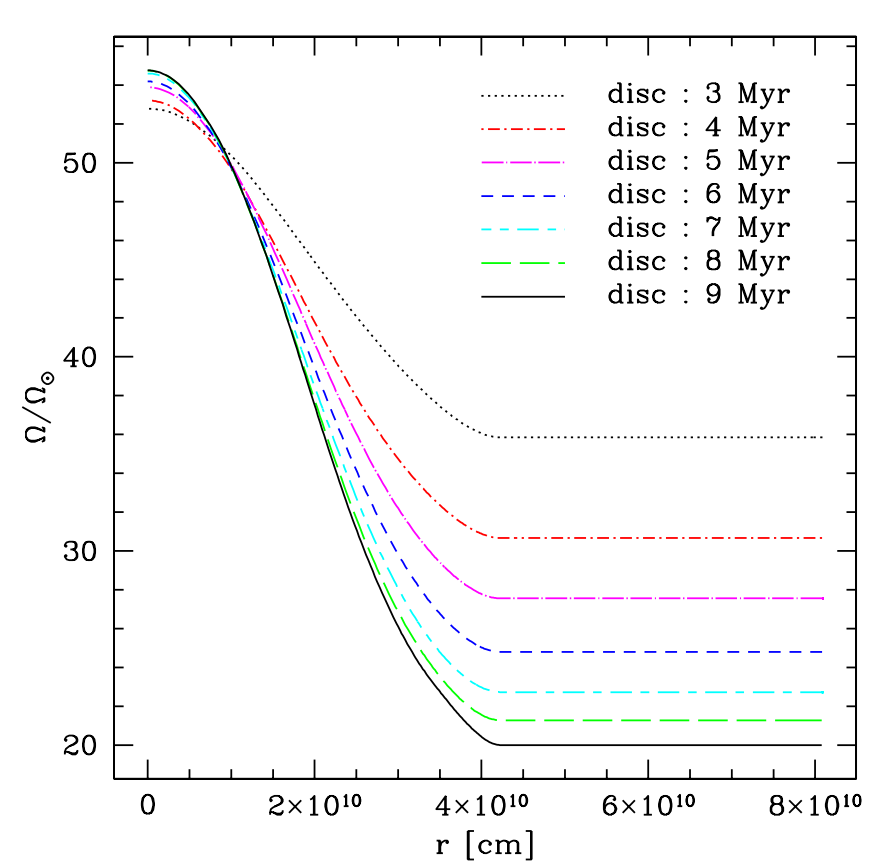

Fig. 9. Rotation profiles at an age of $9 \mathrm{Myr}$ (corresponding to the disappearance of the disc for the model with the longer disc lifetime) for models computed with different disc lifetimes.

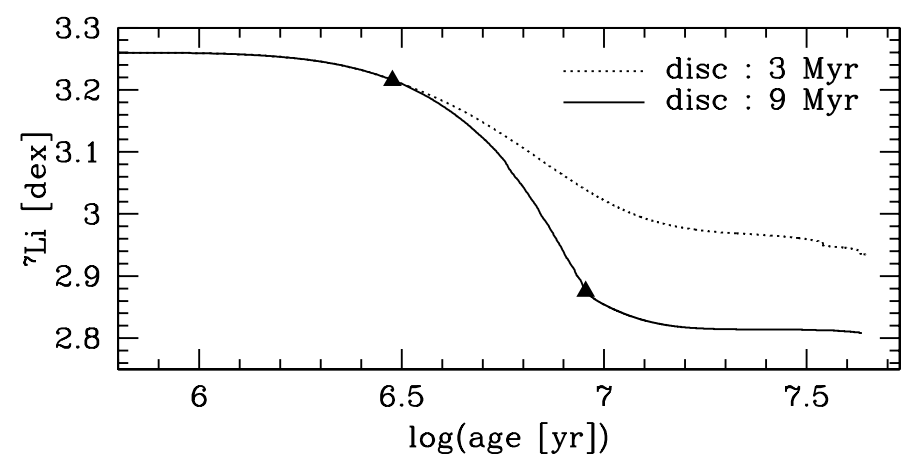

Fig. 10. Evolution of the surface lithium abundance for two $1 M_{\odot}$ models computed with a mixing-length parameter $\alpha=0.6 \alpha_{\odot}$. The continuous and dotted lines correspond to disc lifetimes of 9 and $3 \mathrm{Myr}$, respectively. Triangles indicate the end of the disc-locking phase for each model.

the mixing-length parameter. The PMS evolution of two additional rotating models is then computed with a mixing-length parameter $\alpha=0.6 \alpha_{\odot}$ and two different disc lifetimes of 3 and 9 Myr. Figure 10 shows the evolution of the surface lithium abundance for these models during the PMS. As expected, the reduced value of the mixing-length parameter results in a lower PMS lithium depletion for both models. More importantly, we see that the increased duration of the disc-locking phase also leads to a significant decrease in the lithium abundance on the ZAMS, in good agreement with the results obtained above for solar-calibrated models.

Two different parameters are found to determine the quantitative impact of rotational effects on PMS lithium depletion: the initial rotational velocity and the disc lifetime. For a given disc lifetime but different initial velocities, a direct correlation between the surface velocity and the lithium abundance at the end of the PMS is then obtained so that fast rotators are predicted to

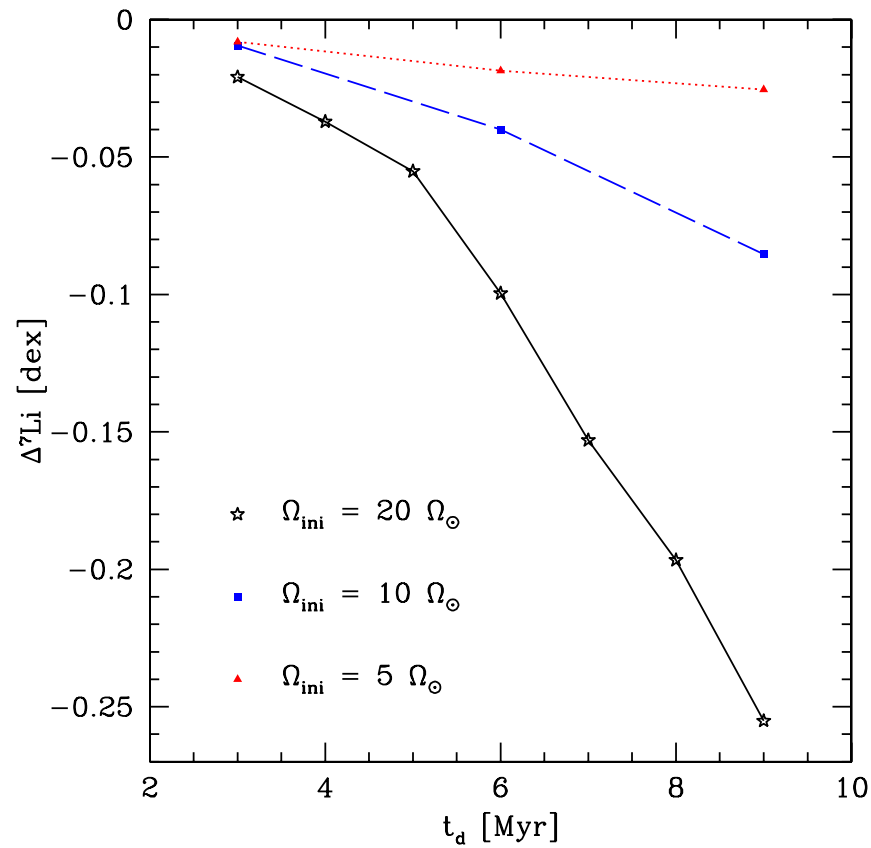

Fig. 11. Surface lithium depletion on the ZAMS for $1 M_{\odot}$ models computed with different disc lifetimes $\left(t_{\mathrm{d}}\right)$ and initial velocities $\left(\Omega_{\text {ini }}\right) . \Delta^{7} \mathrm{Li}$ is defined as the difference between the surface lithium abundance on the ZAMS of rotating models and the non-rotating one.

be more lithium depleted than slow rotators. For a given initial velocity but different disc lifetimes, one expects that slow rotators on the ZAMS are more lithium depleted than fast rotators. This is illustrated in Fig. 11, which shows the lithium depletion on the ZAMS as a function of the disc lifetime for rotating models computed with different initial velocities. We see that models with longer disc lifetimes, hence lower rotation rates on the ZAMS, exhibit higher lithium depletions than models with shorter disc lifetimes that are fast rotators on the ZAMS. This agrees well with observations in the Pleiades of higher lithium abundances for fast rotators than for slow rotators (Soderblom et al. 1993). Moreover, longer disc lifetimes may favour the formation and migration of giant exoplanets, so that lower lithium abundance for exoplanet-host stars are also predicted in this context. This agrees well with the possible detection of differences in the lithium content of stars with and without exoplanets reported by Israelian et al. (2009) (but see also Baumann et al. 2010, for a contrary view).

We may wonder whether the results obtained here in the context of PMS models including shellular rotation are purely academic, given the need of another mechanism for the transport of angular momentum to account for the solar rotation profile for instance (e.g. Mestel \& Weiss 1987; Charbonneau \& MacGregor 1993; Eggenberger et al. 2005; Denissenkov 2010). The rotational periods of young solar-type stars suggest that slow rotators develop a high degree of differential rotation between the radiative core and the convective envelope (see e.g. Bouvier 2008; Denissenkov et al. 2010). This indicates that the hypothesis of shellular rotation during the PMS evolution is perfectly valid in this case even if an additional process can eventually intervene later on during the main-sequence evolution, leading to a flat rotation profile at the solar age.

For fast rotators, the situation is somewhat different. The observation of rotational periods of young solar-type stars suggests that an efficient mechanism for the transport of angular 
momentum is needed for fast rotators to correctly reproduce their spin-down in open clusters (e.g. Bouvier 2008; Denissenkov et al. 2010). The hypothesis of shellular rotation during the PMS thus seems more questionable in the case of fast rotators than for slow rotators. This hypothesis is however not incompatible with the observation of rotational periods for fast rotating solar-type stars, because the strong internal coupling suggested by these observations is not needed before the beginning of the main sequence. This means that a fast rotator can exhibit differential rotation during the PMS with an additional mechanism for the transport of angular momentum at work with a short coupling timescale, leading to an approximate flat rotation profile at the end of the PMS. Moreover, Fig. 8 shows that the effects of rotation and disc lifetime on lithium depletion occur mainly during the first $10 \mathrm{Myr}$ of the PMS evolution. The results obtained in this study will then remain qualitatively valid even in the case of a fast rotator that only exhibits differential rotation during the first $10 \mathrm{Myr}$ and then rotates approximately as a solid-body during the rest of its PMS evolution. We thus conclude that even if the physical mechanisms responsible for the transport of angular momentum in the interiors of solar-type stars are currently not fully understood, the assumption of shellular rotation during the PMS seems to be reasonable according to current observational constraints.

We note that including a mechanism that enforces solid-body rotation during the whole PMS evolution will change the picture. In the case of solar-type stars, the efficiency of rotational mixing is indeed strongly reduced when solid-body rotation is imposed (see for more details Sect. 3 of Eggenberger et al. 2010b). The effects of rotational mixing then become negligible compared to hydrostatic effects of rotation. As a result, the impact of disc lifetime on lithium depletion differs from the one described above. For PMS models in solid-body rotation, a longer disc lifetime reduces the rotational velocity of the star, which simply results in a decrease in the hydrostatic effects of rotation (without any increase in the efficiency of rotational mixing), hence to an overall decrease in the impact of rotation on lithium depletion. The relationship between slow rotation and low lithium abundance on the ZAMS is then found to disappear when solid-body rotation is enforced during the PMS.

\section{Conclusions}

The effects of rotation on the PMS surface lithium abundance of solar-type stars have been studied. Accounting for rotational effects results in an overall decrease in the lithium content at the end of the PMS. The effects of rotation on the PMS lithium content of solar-type stars are enhanced when the initial velocity of the star increases. Moreover, the impact of rotation on PMS lithium depletion is found to depend on the duration of the disc-locking phase, during which the surface angular velocity of the star remains constant. When the disc lifetime increases, the PMS lithium abundance of a solar-type star is indeed found to decrease. This is due to the increase in the efficiency of rotational mixing, which is related to the larger amount of differential rotation generated in the radiative zone during the disc-locking phase. This illustrates that the efficiency of shear mixing is more direcly related to differential rotation in stellar interiors than to surface rotational velocities, as also found in the case of stars undergoing magnetic braking (e.g. Meynet et al. 2011).

A link between the surface velocity and lithium abundance at the end of the PMS is then obtained: slow rotators on the ZAMS are predicted to be more lithium depleted than fast rotators due to the increase in the disc lifetime. This relationship is particularly interesting in the context of the observation of higher lithium abundances for fast rotators than for slow rotators in the Pleiades (Soderblom et al. 1993) and of the possible detection of differences in the lithium content of stars with and without exoplanets reported by Israelian et al. (2009); but see also Baumann et al. (2010) for a contrasting view. The exact degree of coupling between the star and its disc may of course differ from the simple assumption of disc-locking used here, while the transport of angular momentum in the radiative zone of PMS solar-type stars may be influenced by additional physical processes such as magnetic fields. We are indeed still far from having a complete understanding of the various physical processes at work during the evolution of solar-type stars, but this study interestingly illustrates that the impact of rotational mixing depends on the interaction between the star and its disc.

Acknowledgements. Part of this work was supported by the Swiss National Science Foundation.

\section{References}

Asplund, M., Grevesse, N., Sauval, A. J., \& Scott, P. 2009, ARA\&A, 47, 481 Baumann, P., Ramírez, I., Meléndez, J., Asplund, M., \& Lind, K. 2010, A\&A, 519, A87

Bouvier, J. 2008, A\&A, 489, L53

Chaboyer, B., \& Zahn, J.-P. 1992, A\&A, 253, 173

Charbonneau, P., \& MacGregor, K. B. 1993, ApJ, 417, 762

D'Antona, F., \& Mazzitelli, I. 1994, ApJS, 90, 467

D’Antona, F., \& Montalbán, J. 2003, A\&A, 412, 213

Denissenkov, P. A. 2010, ApJ, 719, 28

Denissenkov, P. A., Pinsonneault, M., Terndrup, D. M., \& Newsham, G. 2010, ApJ, 716, 1269

Eggenberger, P., Maeder, A., \& Meynet, G. 2005, A\&A, 440, L9

Eggenberger, P., Meynet, G., Maeder, A., et al. 2008, Ap\&SS, 316, 43

Eggenberger, P., Maeder, A., \& Meynet, G. 2010a, A\&A, 519, L2

Eggenberger, P., Meynet, G., Maeder, A., et al. 2010b, A\&A, 519, A116

Grevesse, N., \& Noels, A. 1993, in Origin and evolution of the elements: proceedings of a symposium in honour of H. Reeves, held in Paris, June 2225, 1992, ed. N. Prantzos, E. Vangioni-Flam, \& M. Casse (Cambridge, England: Cambridge University Press), 14

Israelian, G., Delgado Mena, E., Santos, N. C., et al. 2009, Nature, 462, 189

Maeder, A. 2009, Physics, Formation and Evolution of Rotating Stars (Berlin Heidelberg: Springer)

Maeder, A., \& Meynet, G. 2001, A\&A, 373, 555

Maeder, A., \& Zahn, J.-P. 1998, A\&A, 334, 1000

Martin, E. L., \& Claret, A. 1996, A\&A, 306, 408

Mendes, L. T. S., D’Antona, F., \& Mazzitelli, I. 1999, A\&A, 341, 174

Mestel, L., \& Weiss, N. O. 1987, MNRAS, 226, 123

Meynet, G., Eggenberger, P., \& Maeder, A. 2011, A\&A, 525, L11

Piau, L., \& Turck-Chièze, S. 2002, ApJ, 566, 419

Pinsonneault, M. H. 2010, in IAU Symp. 268, ed. C. Charbonnel, M. Tosi, F. Primas, \& C. Chiappini, 375

Pinsonneault, M. H., Kawaler, S. D., Sofia, S., \& Demarque, P. 1989, ApJ, 338, 424

Pinsonneault, M. H., Kawaler, S. D., \& Demarque, P. 1990, ApJS, 74, 501

Schlattl, H., \& Weiss, A. 1999, A\&A, 347, 272

Soderblom, D. R., Jones, B. F., Balachandran, S., et al. 1993, AJ, 106, 1059

Ventura, P., Zeppieri, A., Mazzitelli, I., \& D’Antona, F. 1998, A\&A, 331, 1011

Zahn, J.-P. 1992, A\&A, 265, 115 